\title{
From neurology to oncology: what have in common autism and cancer? the role of oncogenes, immune system and microbiota
}

\begin{abstract}
Autism and cancer are the products of complex interactions between genetic and environmental factors, for the most part yet unclarified in autism, better characterized in cancer. Even though autism and cancer may appear as two separate conditions with nothing in common, they share a conspicuous number of similarities at the level of pathogenesis that may be useful for translating preventive and therapeutic approaches from one disease to the other. Oncogene-associated signaling appears to play an adaptive or compensatory role in autism in contrast with cancer where it is one of the culprits for initiation of the disease. Immunological deficiencies and chronic inflammation are present in both diseases, and approaches aimed at balancing the immune system and reducing inflammation are useful in prevention and treatment of both. Alterations of the microbiota have a causative role in autism whereas in cancer it is yet to be determined whether they are causes or effects of the disease.
\end{abstract}

Volume 8 Issue 3 - 2018

\author{
Marco Ruggiero,' Stefania Pacini \\ 'Molecular biology, Retired full professor of molecular biology \\ at the University of Firenze, Italy \\ ${ }^{2}$ Biological sciences, Retired associated professor of human \\ anatomy at the University of Firenze, Italy
}

\begin{abstract}
Correspondence: Marco Ruggiero, Silver Spring Sagl,Via Raimondo Rossi 24,Arzo-Mendrisio 6864, Switzerland,Tel +4l 79230 9283, Email marco.drruggiero@gmail.com,
\end{abstract} pacini68@yahoo.com

Received: March 20, 2018| Published: May 25, 2018

Keywords: autism, cancer, oncogenes, immune system, microbiota

\section{Introduction}

At first glance autism and cancer appear to be unrelated conditions with little if anything in common. According to the fifth edition of the Diagnostic and Statistical Manual of Mental Disorders (DSM-5), autism spectrum disorders are defined as a set of symptoms such as:

Persistent deficits in social communication and social interaction across multiple contexts, as manifested by the following, currently or by history:

i. Deficits in social-emotional reciprocity, ranging, for example, from abnormal social approach and failure of normal back-andforth conversation; to reduced sharing of interests, emotions, or affect; to failure to initiate or respond to social interactions.

ii. Deficits in nonverbal communicative behaviors used for social interaction, ranging, for example, from poorly integrated verbal and nonverbal communication; to abnormalities in eye contact and body language or deficits in understanding and use of gestures; to a total lack of facial expressions and nonverbal communication.

iii. Deficits in developing, maintaining, and understand relationships, ranging, for example, from difficulties adjusting behavior to suit various social contexts; to difficulties in sharing imaginative play or in making friends; to absence of interest in peers. ${ }^{1}$

There exist many definitions of cancer but they all agree on the principle that "Cancer is not just one disease, but a large group of almost 100 diseases. Its two main characteristics are uncontrolled growth of the cells in the human body and the ability of these cells to migrate from the original site and spread to distant sites. If the spread is not controlled, cancer can result in death."

Comparison between the criteria for autism and cancer diagnosis evidences that autism is defined by a set of non-objective symptoms, whereas cancer is defined by a number of objective criteria ranging from histology to molecular biology. In addition, if the criteria for the diagnosis of cancer are relatively straightforward and there is national and international consensus on the assessment of severity and progression of the disease through staging and grading, the diagnosis of autism is still subjected to periodical revisions and, in the United States, there is not even uniformity in the criteria for diagnosis between the different States of the Union. ${ }^{3}$ Also, the interest for the two diseases appears to be very different and such a difference is present both in the general public and specialized researchers. Thus, the search engine Google Trends evidences that the interest over time for searches for "autism" is, on average, 8 , whereas the interest for cancer is 72 , and this trend appears to be rather constant since the inception of the system in the year 2004 (according to Google, "the numbers for 'interest over time' represent search interest relative to the highest point on the chart for the given region and time. A value of 100 is the peak popularity for the term. A value of 50 means that the term is half as popular. Likewise, a score of zero means the term was less than $1 \%$ as popular as the peak"). In the area of peer-reviewed papers retrievable from the database PubMed, such a difference is even more evident. A search for "autism" performed at the end of 2017 yielded about 40,000 papers with an exponential rise in the past 17 years. A search for "cancer" performed the same day, yielded about 3.5 million papers with a steady growth for the past 40 years. These simple observation may be interpreted in a number of ways that are not necessarily mutually exclusive.

i. Autism was relatively rare until two decades ago whereas cancer has been recognized as a major threat to public health since, at least, the end of World War Two.

ii. Research on cancer has received many more funds in the past decades in comparison with research on autism.

iii. Public awareness of autism is relatively recent whereas awareness of cancer is decade-old.

iv. Because of uncertainties on the criteria of diagnosis, it is plausible 
that the prevalence of autism is underestimated whereas that of cancer is probably much more accurate.

Whatever the case, it is safe to state that the research on cancer has produced many more results than that on autism with tremendous achievements in knowledge of the diseases, epidemiology, prevention, early diagnosis, therapy and prognosis. Conversely, in the field of autism, since the etiology of the disease is still unknown, there is no consensus on ways to prevent the condition, there is no consensus on therapy and the evolution of the disease and its prognosis are not predictable. It appears that these two areas of medicine are very distant from whichever point of view one wishes to consider them.

However, as odd as it may seem, a PubMed search for the two words "autism and cancer" in the same document, yielded 972 papers, a number that, although being a minor fraction of the papers on each individual subject, still represents a significant figure. Although most of the papers retrieved in this search are only marginally related to the interconnections between autism and cancer, nevertheless there are studies pointing to common traits between the two disease. For example, a recent study exploring the epidemiological correlation between rates of cancer and autism concluded that "... there may be an association between autism and specific forms of cancer" (3), and another study "demonstrated that patients with autistic disorder have an increased risk of cancer." "The scope of this review is to evidence similarities and analogies between autism and cancer with the hope that the significant results obtained in oncology may somehow be transferred to the field of autism. In our opinion, there are three areas where autism and cancer share similarities and they consist in the involvement of:

i. Inter- and intracellular signaling.

ii. The immune system.

iii. The microbiota

\section{Similarities and analogies in autism and cancer cell signaling}

One of the major achievements in cancer research was represented by the characterization of the molecular hallmarks of cancer that begun with the discovery of cancer-causing genes (oncogenes), soon followed by the discovery of tumor suppressing genes - originally called anti-oncogenes - as well as by a number of other cancer-related genes responsible for a number of features of cancer cells. ${ }^{5}$ It is now evident that all genes involved in cancer onset and progression also play a physiological role in the most fundamental functions of normal cells such as proliferation, differentiation and apoptosis. It is also evident that many of the genes involved in cancer play their role as elements of inter- and intracellular signaling; since one of the hypotheses about autism pathogenesis concerns altered brain connectivity, ${ }^{6}$ and since inter- and intracellular signaling are essential for brain connectivity, it is reasonable that genes originally studied for their association with cancer may also be associated with autism. The following examples show the involvement of the products of some representative proto-oncogenes in autism.

Platelet-Derived Growth Factor (PDGF), the product of the simian sarcoma virus transforming gene, $\mathrm{p} 28$ sis and one the first examples of autocrinicity, ${ }^{7}$ is increased in the serum of autistic children compared to healthy age- and gender-matched subjects as demonstrated by a study performed in Japan in $2010 .{ }^{8}$ More specifically, in this study, a significant and positive correlation between the serum levels of the BB homodimer of PDGF and the Autism Diagnostic InterviewRevised domain $\mathrm{C}$ scores that evaluate stereotyped patterns of behavior, was observed. The correlation appeared to be specific for PDGF-BB as no correlation with serum levels of PDGF-AA, PDGF $\mathrm{AB}$ or VEGF (Vascular Endothelial Growth Factor) were observed. A study performed two years later in Saudi Arabia, confirmed these observation, noting that the levels of PDGF, but not those of VEGF, were significantly higher in autistic children compared to neurotypical subjects and the increase was significantly more pronounced in children with severe autism as compared with children with milder symptoms. ${ }^{9}$ It is worth noticing that a more recent study conducted in 2017 in Australia, yielded opposite results, with reduced levels of PDGF-BB associated with increased severity of autistic symptomatology both in males and females, in a large cohort of 144 children diagnosed with autism. ${ }^{10}$ The reasons for this discrepancy have not been elucidated; one hypothesis may be related to the peculiar age-related pattern of expression of the growth factor, with healthy children between ages 7 to 17 showing higher serum levels of PDGF-BB compared to younger children and adults. ${ }^{11}$ A single observation in an animal experimental model seems to corroborate the hypothesis that deficient PDGF signaling is associated with autism behaviors; PDGF receptor- $\beta$ gene knockout mice show deficits in cognitive and socio-emotional areas that are considered representative for some of the symptoms in autistic and schizophrenic patients. ${ }^{12}$ Since PDGF, in addition to being a potent mitogen, is also fundamental in development of the central nervous system, ${ }^{13}$ deficits of PDGF-associated signaling may be associated with altered development and connectivity, and elevated serum levels of PDGF may represent a compensatory mechanism. It is worth mentioning that the papers on PDGF and cancer retrievable in PubMed is over 3,000, whereas the papers on PDGF and autism are less than 10; it may thus be assumed that as the number of studies on the association between PDGF signaling and autism increases, the discrepancies quoted above will be solved and the role of this signaling pathway in autism will be clarified.

Another oncogene-related signaling pathway widely studied in cancer that is gaining attention in autism is represented by the pathway pertaining to the Epidermal Growth Factor (EGF) and its receptor. The reason for interest in EGF in autism has to be found in the observation that in healthy individuals it has key roles in the growth, proliferation, and differentiation of numerous cell types, and is involved in several pathways pertaining to neuronal function. ${ }^{14-16}$

As it happens with PDGF, there are studies reporting that children with autism have decreased plasma levels of EGF, ${ }^{17}$ alongside with studies reporting opposite results (i.e. increased EGF levels in autism) and suggesting that "increased levels of EGF might have an importance in the pathophysiology of autism. ${ }^{18,19}$ "According to this latter study conducted in the US in 2017, increased levels of serum EGF may be interpreted as indication of dysregulation of growth and modulatory pathways, rather than of systemic inflammatory responses. Technical problems may account for these discording results; it is possible that EGF, together with other growth factors, including PDGF, may be released by platelets during the blood sampling processing for obtaining serum, thus confounding the results. ${ }^{20}$ It is worth noticing, however, that also the receptor for EGF appears to be elevated in the plasma of autistic subjects and these elevated levels correlate with the severity of symptoms. ${ }^{21}$ According to this study, the levels of EGF receptor correlated with those of Hepatocyte Growth Factor (HGF) and high-mobility group protein B1 (HMGB1), but not of other tested 
biomarkers, thus leading to the conclusion that "A strong correlation between plasma EGFR and HGF and HMGB1 suggests that increased EGFR levels may be associated with the HGF/Met signaling pathway, as well as inflammation. ${ }^{21}$ "

Moving from growth factors and their receptors to downstream signaling proteins such as GTP-binding proteins, other connections between proto-oncogenes and autism can be observed. A notable example is represented by ras signaling, whose involvement in autism was observed in 1993 by French researchers who were looking for associations between autism and genes coding for enzymes involved in monoaminergic metabolism and for a linked marker, c-HarveyRas-1 (HRAS1). These Authors did not observe any association between autism and genes coding for tyrosine hydroxylase, dopamine-beta-hydroxylase (DBH), and tryptophan hydroxylase, but found instead a positive association between autism and the locus containing the gene for HRAS- $1 .{ }^{22}$ Since the Ras-MAPK and PI3KAKT-mTOR pathways play a significant role in neural development and plasticity, in addition to roles in cancer signaling, it can be argued that alterations of these pathways may be associated with onset and development of neurodevelopmental disorders. ${ }^{23}$ Such a hypothesis is corroborated by the recent finding that a high prevalence of autism is present in rare disorders of the Ras/MAPK pathway, namely Noonan, cardiofaciocutaneous (CFC), and Costello syndromes. ${ }^{24}$

As with PDGF- and EGF-related signaling, however, it is not clear whether autism is associated with a decreased function of the ras signaling pathway as suggested by Tylee et al., ${ }^{23}$ or with an increased function as suggested by the association with Noonan and Costello syndromes. ${ }^{24}$

A hypothesis that may help interpreting these apparently contradicting observation consists in highlighting the fragile nature of brain development. Assuming that signaling pathways originally identified as related to cancer onset and development also play a role in physiological brain development, it may be argued that any variation from the norm, that is either increased or decreased function, leads to delays or alterations of brain development that manifest themselves as autism symptoms.

Other examples seem to support this interpretation. For example, a study published in 2010, demonstrated that the expression of the anti-apoptotic gene Bcl2 was significantly decreased in the brains of autistic children, possibly as consequence of decreased expression and phosphorylation/activation of Akt kinase that regulates $\mathrm{Bcl} 2$. At the same time, expression of the tumor suppressor gene $\mathrm{p} 53$, "the guardian of the genome" was increased as compared to brains of age-matched neurotypical subjects. ${ }^{25}$ According to the Authors' interpretation, these alterations suggested that "down-regulation of the BDNF-AktBcl2 anti-apoptotic signaling pathway in the autistic brain could be one of the underlying mechanisms responsible for the pathogenesis of autism. ${ }^{25}$ " Involvement of $\mathrm{Bcl} 2$ may help explaining the therapeutic effects of ketogenic diets in autism. It is well accepted that ketogenic diets are helpful in cancer, ${ }^{26}$ and recent evidence points to their usefulness in autism as well. ${ }^{27}$ An interesting experimental model that is particularly responsive to ketogenic diets is represented by the $\mathrm{BTBRT}+\mathrm{tf} / \mathrm{j}$ (BTBR) mouse, which shows phenotypic characteristics of autism spectrum disorders. In this model it was recently observed that ketogenic diet was associated with increased expression of a member of the Bcl2 family, the BNIP3 BCL2/adenovirus E1B $19 \mathrm{kd}-$ interacting protein 3 (BNIP3). Increased expression of BNIP3 in the liver of animals fed ketogenic diet suggested the potential activation of mitophagy and increased mitochondrial turnover. According to a recent review on mitophagy and cancer, mitophagy, that is a selective mode of autophagy in which mitochondria are specifically targeted for degradation, is deficient in cancer and such defect is associated with "mitochondrial dysfunction that can affect metabolic reprogramming in response to stress, alter cell fate determination and differentiation, which in turn affects disease incidence and etiology, including cancer. ${ }^{28}$ "

Since mitochondrial dysfunction is involved in autism as well, ${ }^{29}$ it can be hypothesized that activation of BNIP3 signaling with consequent increase in mitochondria turnover is the mechanism, common to cancer and autism, through which ketogenic diets are beneficial in both conditions. Albeit indirectly, Bcl2 appears to be an example where information from cancer- and autism-associated signaling converge and may lead to efficient therapeutic approaches.

Involvement of signaling pathways that can be defined as oncogeneassociated in a non-neoplastic conditions such as autism raises an interesting question; are autistic subjects more at risk for cancer? As with other instances quoted above, the answer is contradictory. As an example of these conflicting results, we quote two recent studies. According to a study published in 2010 by Authors from the Department of Psychiatry and Human Behavior of Brown University (Providence, Rhode Island, USA), there are statistically significant correlations between autism rates and the incidence of in situ breast cancer, but not of other neoplasms. The Authors interpreted such a correlation as a consequence of the "high rates of genomic aberrations, including chromosomal rearrangements and de novo copy-number variations" observed in autism, noting that "These observations are reminiscent of cancer, a disease where genomic rearrangements also play a role." "Conversely, a study published in 2016 by Authors from Iowa University (Iowa City, USA), aptly entitled "Autism linked to increased oncogene mutations but decreased cancer rate" concluded that "children and adults with ASD (Autism Spectrum Disorders) exhibited a protective effect against cancer" and "Both males and females demonstrated the protective effect. ${ }^{30}$ ",

It is undeniably difficult to draw conclusions from the few heterogeneous studies on oncogenes and oncogene-related signaling and autism. The prevalent impression is that altered signaling in autism is more of a consequence than a cause for the disease; it may represent a sort of a compensatory response to alterations of neurodevelopment that may be caused by complex interactions between genetic and environmental factors. Nonetheless, as the example of ketogenic diet, $\mathrm{Bcl} 2$ and mitophagy demonstrates, knowledge accumulated in the past 40 years in the molecular biology of cancer can be applied to the emerging field of molecular biology of autism, where it may lead to new hypotheses about etiology and pathogenesis and, more importantly, to effective therapeutic approaches.

\section{Alterations of the immune system in autism and cancer}

Although the terms immunotherapy and oncoimmunology are relatively recent, the origin of the concepts underlying such terms is almost two century old and can be traced back to the times of Dr. William Coley who successfully treated advanced cancers in New York in the second half of the Nineteenth Century. The idea of empowering the immune system to fight cancer is now so widespread that it is even used in commercials, and "Immunotherapy of Cancer" was designated "Breakthrough of the Year 2013" by the Journal Science 
which dedicated to this topic the cover of the December 20 issue. $^{31}$ There exist many forms of cancer immunotherapy that try to exploit both the innate and the adaptive arms of the immune system as well as the ability of targeted antibodies to neutralize cancer-associated proteins, many of which are involved in transmembrane signaling. The whole concept of cancer immunotherapy is based on the observation that cancer cells develop the ability to escape immune surveillance by making themselves "invisible" to the immune system through a variety of mechanisms that evolve as cancer progresses. Hence the need to "re-educate" the immune system to recognize specific cancer antigens and to use antibodies that confer a sort of passive immunity targeting specific cancer proteins. All this is made possible by detailed knowledge of the alterations of the immune system in cancer as well as of the peculiar features of cancer cells as far as their immunogenicity is concerned. Regrettably, such a wealth of knowledge is not present in the field of autism even though an involvement of the immune system in the pathogenesis of the disease is hypothesized by many. ${ }^{32}$ However, from the information that can be retrieved on this topic, it appears that cancer and autism share some common alterations in immune system function, notably in cell-mediated immune responses that seem to be relatively deficient in both diseases. For example, the activity of Natural Killer (NK) cells assessed in 1,027 blood samples from autistic children obtained from ten clinics, was significantly lower compared to that of 113 healthy controls..$^{33}$ Since NK cells play a key role in cancer immune surveillance, and since clinical trials suggest how NK cell-based strategies represent a promising approach to immunotherapy of cancer, ${ }^{34}$ it is hoped for that knowledge gained in NK cell-based immunotherapy of cancer can be translated to therapeutic approaches to autism.

Another type of cells of the innate immune system that appear to play a role both in autism and cancer is represented by mononuclear phagocytes, namely microglial cells in autism, and bone fide macrophages in cancer. Microglial cells are considered essential for the development of normal neural networks and alterations of microglia in autism has been reported by a number of Authors, thus hypothesizing that glial dysfunctions directly contribute to pathophysiology of autism. ${ }^{35,36}$ The role of macrophages in cancer is rather complex as these cells may participate in the fight of the immune system against cancer, but may also be co-opted, together with other cells of the immune system, by cancer cells to favor tumor progression, angiogenesis, cancer-associated immunosuppression and metastases up to the point of being defined "villains and heroes. ${ }^{37}$ " Whatever the case, it seems that immunotherapeutic approaches targeting macrophages yield good symptomatic results in autism and cancer. $^{38,39}$

The role of macrophages and microglial cells can also be interpreted in the context of local and systemic inflammation that constitute hallmarks of autism and cancer with neuro-inflammation being a common trait in autism and other neuro-psychiatric conditions. ${ }^{40}$ It is well known that inflammation is a risk factor and acts as a promoter in cancer, and, therefore, interventions aimed at reducing inflammation and anti-inflammatory drugs yield encouraging results. ${ }^{41}$ Likewise, aberrant activation of microglial cells, the inflammatory brain resident myeloid cells, is considered a key factor in the pathogenesis of autism, and there is convincing evidence indicating that approaches aimed at reducing neuro-inflammation ameliorate the symptoms of autism in experimental models. ${ }^{42}$
Another element that appears to be common in autism and cancer, as far as immune dysregulation is concerned, is represented by alterations of the vitamin D axis. The vitamin D axis is constituted by vitamin $\mathrm{D}$ and its binding proteins that are the carrier protein (Gc-protein) and the nuclear receptor (VDR), both polymorphic in nature. ${ }^{43}$ Association between low serum levels of vitamin D3 and increased risk has been reported for both diseases. ${ }^{44,45}$ with consequent recommendations to supplement the diet of autistic and cancer patients with vitamin D3 when serum levels are low. ${ }^{46,47}$ Deficient signaling through VDR may also play a role as autism and cancer patients share polymorphisms of the gene coding for VDR with the polymorphisms associated with increased cancer risk implicated in autism as well. ${ }^{48,49}$ It can be hypothesized that decreased vitamin D signaling occurring as consequence of low serum levels of the vitamin or because deficient signal transduction through polymorphic VDR, is associated with immune system dysfunction and chronic inflammation that in turn are considered pathogenetic factors for both conditions. Consistent with these observation, immunotherapeutic approaches based on glycosaminoglycans and vitamin D3, targeting inflammation and the immune system are associated with positive outcomes both in cancer and autism. ${ }^{50}$

\section{The role of the microbiota in autism and cancer}

Discovery of the role of the microbiota in health and disease represents a modern paradigm shift as microbes are no longer seen as enemies to be exterminated but rather as friends contributing to our well-being. Bacteria, yeasts, fungi, archaea, protozoa, and viruses are present in every tissue of our bodies, from the gut, where they have been always recognized, to the brain, an organ that was supposed to be sterile until very recently. ${ }^{51}$ As the field is rather new, at variance with other topics of research such as oncogenes, the number of papers on microbiota and cancer and on microbiota and autism is of the same order of magnitude and there is general consensus that healthy microbiota play an important role in prevention of autism and cancer. In autism, the role of the microbiota is interpreted in the context of the so-called gut-brain axis, a concept that led to coining new words such as "neuromicrobiology 52 " or "psychobiotics. ${ }^{53}$ " According to these interpretations, gut microbes are involved in complex host behaviors and brain development and, since alterations of the gut microbiota are common in autism, ${ }^{54}$ it is postulated that there is a bi-directional communication pathway between the gut and the brain with microbes in the gut providing a wealth of information that influences brain development and function. ${ }^{55}$

In addition to the microbes in the gut, it was recently hypothesized that also the microbes resident in the brain may play a role in autism as well as in other neuro-psychiatric disorders. ${ }^{56}$ As far as the role of microbiota in cancer is concerned, it appears that local alterations of microbial populations are associated with cancers arising in specific sites. A recent meta-analysis of studies on this topic, reports that alterations of the colorectal, gastric and esophageal microbiota are associated with cancer development in these sites. Consistent with these observation, it has been hypothesized that microbes pertaining to the cervical microbiota may modify the cytokine profile of the cervical microenvironment during the development of pre-neoplastic lesions and cancer of the cervix. ${ }^{57}$

As the microbiota is essential for development and function of the immune system, ${ }^{58}$ we can envisage at least two types of effects due to alterations of the microbiota in autism or cancer. On one side, 
systemic alterations of the microbiota may lead to impaired function of the immune system and such impairment reflects negatively both in autism development and in cancer progression. On the other side, localized alterations of the microbiota, as they may occur in the colon, stomach, esophagus and cervix for cancer, or in the gut and the brain for autism, may be associated with aberrant local production of cytokines and localized inflammation that is detrimental in both conditions. Consistent with these observation, restoration of microbiota through the administration of ad hoc formulated probiotics has proven useful in both diseases. . $^{59,60}$

It is worth noticing that all the studies quoted so far describe associations between alterations of the microbiota and autism or cancer, and it is well known that association does not mean causation; on the contrary, it may be easily argued that, being autism and cancer complex chronic conditions involving the entirety of the organism, alterations of microbiota could be consequences rather than causes of the diseases. At variance with other noxae, however, in the case of the microbiota it is relatively easy to manipulate the microbial composition through administration of antibiotics or probiotics and, therefore, establish a cause and effect relationship between microbes and diseases. Studies on animals and observation in children seem to indicate that transplantation of gut microbiota is responsible for changes in behavioral phenotypes as if they could be transferred from one individual to another together with the microbes, thus hypothesizing a causal relationship between microbes and behavior. ${ }^{61,62}$ Likewise, preclinical studies in the experimental animal suggest that specific microbial strains are responsible for susceptibility to initiation and to progression of cancer by interfering with the mechanisms of inflammation, DNA damage and restoration, and by producing metabolites that may either facilitate or prevent tumorigenesis. ${ }^{63}$

Given these encouraging results, and considering that fermented foods that today would be called probiotics, were the first processed foods eaten by humans, it is consequential that specific probiotic formulas are being developed with the goal of targeting diseases such as autism and cancer where alterations of the microbiota have been described. ${ }^{64}$

Interestingly, and consistent with the hypotheses put forward above, certain strains such as Lactobacilli and Bifidobacteria appear to be beneficial in both diseases. ${ }^{59,65}$ In addition to their involvement in rebalancing the healthy microbiota and the immune system, other mechanisms of action can be postulated for these beneficial effects in such diverse conditions. For example, the ability of probiotic strains to bind and eliminate mutagenic and neuro-toxic lipophilic toxicants may prove useful in both conditions. ${ }^{66}$ Also the endogenous production of butyrate and acetate may prove useful in both conditions considering that butyrate has proven anti-cancer and neuro-protective properties, and acetate is instrumental for inducing and maintaining a level of ketosis that has proven beneficial in autism and cancer. ${ }^{67-69}$

\section{Conclusion}

We are very well aware that the word "cancer" comprises hundreds of different diseases, each one with different etiology, pathogenesis, prognosis and targeted treatment; that each cancer patient, even when histology, grade and stage are the same, may have a different sets of mutations and that, essentially, every cancer is unique. Likewise, we are aware that "autism" is a word comprising a wide spectrum of disorders that differ for symptoms, severity and prognosis. Both autism and cancer are the product of complex interactions between genetic and environmental factors, for the most part yet unclarified in autism, better characterized in cancer. Autism is a disease of development that typically arises in infancy, whereas the majority of cancers occurs in adulthood and later, thus suggesting that even if the same genes may be involved, the pathogenetic mechanisms are different. We know, therefore, that any attempt to reduce autism or cancer to a limited series of definitions or factors is an exercise that not only is futile, but also profoundly flawed. It is not as to compare apples to oranges, but rather as to compare apples to French fries; both are edible, but this may be the only quality they have in common. Nevertheless, as the human mind is trained to find common traits in the attempt to see some order in the universe, we feel that the similarities we have described in autism and cancer may be helpful to understand how the human body and brain react to complex alterations. They may help stimulating interest in the role of the immune system and the microbiota in the pathogenesis of both conditions. Thus, it is well known that signaling pathways pertaining to the microbiota influence neurologic diseases, ${ }^{70}$ and the colon, where the most part of the microbiota resides, may be the key to understand health and disease. ${ }^{71}$ We hope that results, observation, insights gained in one field may be translated to the other with the ultimate goal of helping patients suffering from these diseases.

\section{Significance of work}

In this article, we describe a number of similarities and analogies between autism and cancer that involve oncogene signaling, the immune system and the microbiota. Knowledge of the traits that are common to the two diseases will help understanding how the human body and brain react to complex alterations. They will help stimulating interest in the role of the immune system and the microbiota in the pathogenesis of both conditions. Finally, they will help translating results, observation, insights gained in one field to the other with the ultimate goal of helping patients suffering from these diseases.

\section{Authors' contribution}

The Authors contribute equally to this paper developing the concepts described in this study and writing the manuscript.

\section{Competing interest}

Marco Ruggiero is the founder and CEO of Silver Spring, a Swiss research and development company in the field of supplements and probiotics. Stefania Pacini is a consultant for Silver Spring. Drs. Pacini and Ruggiero consult for several companies producing supplements and other remedies.

\section{Funding}

This study did not receive any specific grant from funding agencies in the public, commercial, or not-for-profit sectors.

\section{References}

1. Diagnostic and Statistical Manual of Mental Disorders. Fifth Edition. VA, Arlington: American psychiatric association; 2013.

2. Gale encyclopedia of medicine; 2008

3. Kao HT, Buka SL, Kelsey KT, et al. The correlation between rates of cancer and autism: an exploratory ecological investigation. PLoS One. 2010;5(2):e9372.

4. Chiang HL, Liu CJ, Hu YW, et al. Risk of cancer in children, adolescents, 
and young adults with autistic disorder. J Pediatr. 2015;166(2):418-423

5. Bertram JS. The molecular biology of cancer. Mol Aspects Med. 2000;21(6):167-223.

6. O'Reilly C, Lewis JD, Elsabbagh M. Is functional brain connectivity atypical in autism? A systematic review of EEG and MEG studies. PLoS One. 2017;12(5):e0175870.

7. Robbins $\mathrm{KC}$, Antoniades $\mathrm{HN}$, Devare SG, et al. Structural and immunological similarities between simian sarcoma virus gene product(s) and human platelet-derived growth factor. Nature. 1983;305(5935):605608

8. Kajizuka M, Miyachi T, Matsuzaki H, et al. Serum levels of plateletderived growth factor $\mathrm{BB}$ homodimers are increased in male children with autism. Prog Neuropsychopharmacol Biol Psychiatry. 2010;34(1):154158

9. Zakareia FA, Al-Ayadhi LY, Al-Drees AA. Study of dual angiogenic/ neurogenic growth factors among Saudi autistic children and their correlation with the severity of this disorder. Neurosciences (Riyadh) 2012;17(3):213-218.

10. Masi A, Breen EJ, Alvares GA, et al. Cytokine levels and associations with symptom severity in male and female children with autism spectrum disorder. Mol Autism. 2017;8:63.

11. Kleiner G, Marcuzzi A, Zanin V, et al. Cytokine levels in the serum of healthy subjects. Mediators Inflamm. 2013; 434010:6.

12. Nguyen PT, Nakamura T, Hori E, et al. Cognitive and socio-emotional deficits in platelet-derived growth factor receptor- $\beta$ gene knockout mice. PLoS One. 2011;6(3):e18004.

13. Funa K, Sasahara M. The roles of PDGF in development and during neurogenesis in the normal and diseased nervous system. J Neuroimmune Pharmacol. 2014;9(2):168-181.

14. Wieduwilt MJ, Moasser MM. The epidermal growth factor receptor family: biology driving targeted therapeutics. Cell Mol Life Sci. 2008;65(10):1566-1584

15. Aguirre A, Dupree JL, Mangin JM, et al. A functional role for EGFR signaling in myelination and remyelination. Nat Neurosci. 2007;10(8):990-1002.

16. Galvez-Contreras AY, Quiñones-Hinojosa A, Gonzalez-Perez O. The role of EGFR and ErbB family related proteins in the oligodendrocyte specification in germinal niches of the adult mammalian brain. Front Cell Neurosci. 2013;7:258.

17. Onore C, Van de Water J, Ashwood P. Decreased levels of EGF in plasma of children with autism spectrum disorder. Autism Res Treat. 2012;2012:205362.

18. Işeri E, Güney E, Ceylan MF, et al. Increased serum levels of epidermal growth factor in children with autism. J Autism Dev Disord. 2011;41(2):237-241.

19. Pardo CA, Farmer CA, Thurm A, et al. Serum and cerebrospinal fluid immune mediators in children with autistic disorder: a longitudinal study. Mol Autism. 2017;8:1

20. Lubkowska A, Dolegowska B, Banfi G. Growth factor content in PRP and their applicability in medicine. $J$ Biol Regul Homeost Agents. 2012;26(2 Suppl 1):3S-22S

21. Russo AJ. Increased epidermal growth factor receptor (EGFR) associated with hepatocyte growth factor (HGF) and symptom severity in children with autism spectrum disorders (ASDs). J Cent Nerv Syst Dis. 2014;6:79-83.

22. Hérault J, Perrot A, Barthélémy C, et al. Possible association of c-HarveyRas-1 (HRAS-1) marker with autism. Psychiatry Res. 1993;46(3):261267.

23. Tylee DS, Hess JL, Quinn TP, et al. Blood transcriptomic comparison of individuals with and without autism spectrum disorder: A combinedsamples mega-analysis. Am J Med Genet B Neuropsychiatr Genet. 2017;174(3):181-201.

24. Garg S, Brooks A, Burns A, et al. Autism spectrum disorder and other neurobehavioural comorbidities in rare disorders of the Ras/MAPK pathway. Dev Med Child Neurol. 2017;59(5):544-549.

25. Sheikh AM, Malik M, Wen G, et al. BDNF-Akt-Bcl2 antiapoptotic signaling pathway is compromised in the brain of autistic subjects. $J$ Neurosci Res. 2010;88(12):2641-2647.

26. Klement RJ. Beneficial effects of ketogenic diets for cancer patients: a realist review with focus on evidence and confirmation. Med Oncol. 2017;34(8):132.

27. Napoli E, Dueñas N, Giulivi C. Potential therapeutic use of the ketogenic diet in autism spectrum disorders. Front Pediatr. 2014;2:69.

28. Drake LE, Springer MZ, Poole LP, et al. Expanding perspectives on the significance of mitophagy in cancer. Semin Cancer Biol. 2017;47:110 124

29. Hollis F, Kanellopoulos AK, Bagni C. Mitochondrial dysfunction in autism spectrum disorder: clinical features and perspectives. Curr Opin Neurobiol. 2017;45:178-187.

30. Darbro BW, Singh R, Zimmerman MB, et al. Autism linked to increased oncogene mutations but decreased cancer rate. PLoS One. 2016;11(3):e0149041.

31. Couzin-Frankel J. Breakthrough of the year 2013. Cancer immunotherapy. Science. 2013;342(6165):1432-1433.

32. Masi A, Glozier N, Dale R, et al. The immune system, cytokines, and biomarkers in autism spectrum disorder. Neurosci Bull. 2017;33(2):194 204.

33. Vojdani A, Mumper E, Granpeesheh D, et al. Low natural killer cell cytotoxic activity in autism: the role of glutathione, IL-2 and IL-15. $J$ Neuroimmunol. 2008;205(1-2):148-154.

34. Cifaldi L, Locatelli F, Marasco E, et al. Boosting natural killer cell based immunotherapy with anticancer drugs: a perspective. Trends Mol Med. 2017;23(12):1156-1175.

35. Edmonson CA, Ziats MN, Rennert OM. A Non-inflammatory role for microglia in autism spectrum disorders. Front Neurol. 2016;7:9.

36. Petrelli F, Pucci L, Bezzi P. Astrocytes and microglia and their potential link with autism spectrum disorders. Front Cell Neurosci. 2016;10:21.

37. Kees T, Egeblad M. Innate immune cells in breast cancer - from villains to heroes? J Mammary Gland Biol Neoplasia. 2011;16(3):189-203.

38. Ruggiero M. Gc Protein-Derived Macrophage Activating Factor (GcMAF) and Autism: Do clinical results require a novel interpretation? Am J Immunol. 2016;12(4):77-82.

39. Saburi E, Saburi A, Ghanei M. Promising role for Gc-MAF in cancer immunotherapy: from bench to bedside. Caspian J Intern Med. 2017;8(4):228-238.

40. Nakagawa Y, Chiba K. Involvement of neuroinflammation during brain development in social cognitive deficits in autism spectrum disorder and schizophrenia. J Pharmacol Exp Ther. 2016;358(3):504-515.

41. Ma J, Cai Z, Wei H, et al. The anti-tumor effect of aspirin: What we know and what we expect. Biomed Pharmacother. 2017;95:656-661.

42. Bhandari R, Kuhad A. Resveratrol suppresses neuroinflammation in the experimental paradigm of autism spectrum disorders. Neurochem Int. 2017 103:8-23.

43. Chishimba L, Thickett DR, Stockley RA, et al. The vitamin D axis in the lung: a key role for vitamin D-binding protein. Thorax. 2010;65(5):456462 .

44. Tostes MH, Polonini HC, Gattaz WF, et al. Low serum levels of 25 
hydroxyvitamin D (25-OHD) in children with autism. Trends Psychiatry Psychother. 2012;34(3):161-163.

45. Fleet JC, DeSmet M, Johnson R, et al. Vitamin D and cancer: a review of molecular mechanisms. Biochem J. 2012;441(1):61-76.

46. Saad K, Abdel-Rahman AA, Elserogy YM, et al. Vitamin D status in autism spectrum disorders and the efficacy of vitamin D supplementation in autistic children. Nutr Neurosci. 2016;19(8):346-351.

47. Gröber U, Kisters K, Adamietz IA. Vitamin D in oncology: Update 2015. Med Monatsschr Pharm. 2015;38(12):512-516.

48. Gandini S, Gnagnarella P, Serrano D, et al. Vitamin D receptor polymorphisms and cancer. Adv Exp Med Biol. 2014;810:69-105.

49. Coşkun S, Şimşek Ş, Camkurt MA, et al. Association of polymorphisms in the vitamin D receptor gene and serum 25-hydroxyvitamin D levels in children with autism spectrum disorder. Gene. 2016;588(2):109-114.

50. Peter T., Ruggiero M. Ketogenic diet and immunotherapy in cancer and neurological diseases. 4th international congress on integrative medicine, 1, 2 April 2017, Fulda, Germany. Am J Immunol. 2017;13(3):158-164.

51. Branton WG, Ellestad KK, Maingat F, et al. Brain microbial populations in HIV/AIDS: $\alpha$-proteobacteria predominate independent of host immune status. PLoS One. 2013;8(1):e54673.

52. de la Fuente-Nunez C, Meneguetti BT, Franco OL, et al. Neuromicrobiology: how microbes influence the brain. ACS Chem Neurosci. 2017;9(2):141-150.

53. Misra S, Mohanty D. Psychobiotics: A new approach for treating mental illness? Crit Rev Food Sci Nutr. 2017;30:1-7.

54. Li Q, Han Y, Dy ABC, et al. The gut microbiota and autism spectrum disorders. Front Cell Neurosci. 2017;11:120.

55. Kelly JR, Minuto C, Cryan JF, et al. Cross talk: the microbiota and neurodevelopmental disorders. Front Neurosci. 2017;11:490.

56. Ruggiero M. Fecal Microbiota Transplantation and the brain microbiota in neurological diseases. Clin Endosc. 2016;49(6):579.

57. Avilés-Jiménez F, Guogin Y, Torres-Poveda K, et al. On the search to elucidate the role of the microbiota in the genesis of cancer: the cases of gastrointestinal and cervical cancer. Arch Med Res. 2017;48(8):754-765.

58. Belkaid Y, Hand TW. Role of the microbiota in immunity and inflammation. Cell. 2001;157(1):121-141.
59. Shaaban SY, El Gendy YG, Mehanna N2, et al. The role of probiotics in children with autism spectrum disorder: A prospective, open-label study. Nutr Neurosci. 2017;7:1-6.

60. Motevaseli E, Dianatpour A, Ghafouri-Fard S. The Role of Probiotics in Cancer Treatment: Emphasis on their In Vivo and In Vitro Anti-metastatic Effects. Int J Mol Cell Med. 2017;6(2):66-76.

61. Cenit MC, Sanz Y, Codoñer-Franch P. Influence of gut microbiota on neuropsychiatric disorders. World J Gastroenterol. 2017;23(30):54865498.

62. Kang DW, Adams JB, Gregory AC, et al. Microbiota transfer therapy alters gut ecosystem and improves gastrointestinal and autism symptoms: an open-label study. Microbiome. 2017;5(1):10.

63. Bhatt AP, Redinbo MR, Bultman SJ. The role of the microbiome in cancer development and therapy. CA Cancer J Clin. 2017;67(4):326-344.

64. Pacini S, Ruggiero M. Description of a novel probiotic concept: implications for the modulation of the immune system. Am J Immunol. 2017;13(2):107-113

65. Chong ES. A potential role of probiotics in colorectal cancer prevention: review of possible mechanisms of action. World J Microbiol Biotechnol. 2014;30(2):351-74.

66. Blythe J, Ruggiero M, Pacini S. Case Report: Intermittent fasting and probiotic yogurt consumption are associated with reduction of serum alpha-N-acetylgalactosaminidase and increased urinary excretion of lipophilic toxicants. Madridge J Immunol. 2017;1(1):23-27.

67. Louis P, Hold GL, Flint HJ. The gut microbiota, bacterial metabolites and colorectal cancer. Nature Rev Microbiol. 2014;12(10):661-672.

68. Bourassa MW, Alim I, Bultman SJ, et al. Butyrate, neuroepigenetics and the gut microbiome: Can a high fiber diet improve brain health? Neurosci Lett. 2016;625(20):56-63.

69. Mac Kay EM, Barnes RH, Carne HO, et al. Ketogenic activity of acetic acid. J Biol Chem. 1940;135:157-163.

70. Cox LM, Weiner HL. Microbiota signaling pathways that influence neurologic disease. Neurotherapeutics. 2018;15(1):135-145.

71. Gonzalez-Correa CA, Mulett-Vásquez E, Miranda DA, et al. The colon revisited or the key to wellness, health and disease. Med Hypotheses. 2017; 108:133-143. 